\title{
Oral versus Nasal Breathing during Moderate to High Intensity Submaximal Aerobic Exercise
}

\author{
Chase O. LaComb \\ Department of Kinesiology and Nutrition Sciences, University of Nevada, Las Vegas \\ 4505 S. Maryland Parkway, Box 453034, Las Vegas, NV, USA \\ Richard D. Tandy \\ Department of Kinesiology and Nutrition Sciences, University of Nevada, Las Vegas \\ 4505 S. Maryland Parkway, Box 453034, Las Vegas, NV, USA \\ E-mail: dick.tandy@unlv.edu \\ Szu Ping Lee \\ Department of Physical Therapy, University of Nevada, Las Vegas \\ 4505 S. Maryland Parkway, Box 453029, Las Vegas, NV, USA \\ E-mail: szu-ping.lee@unlv.edu \\ John C. Young \\ Department of Kinesiology and Nutrition Sciences, University of Nevada, Las Vegas \\ 4505 S. Maryland Parkway, Box 453034, Las Vegas, NV, USA \\ E-mail: jack.young@unlv.edu \\ James W. Navalta (Corresponding author) \\ Department of Kinesiology and Nutrition Sciences, University of Nevada, Las Vegas \\ 4505 S. Maryland Parkway, Box 453034, Las Vegas, NV, USA \\ E-mail: james.navalta@unlv.edu
}

Received: 04-12- 2016

Accepted: 21-01- 2017

Published: 31-01- 2017

doi:10.7575/aiac.ijkss.v.5n.1p.8

URL: http://dx.doi.org/10.7575/aiac.ijkss.v.5n.1p.8

\begin{abstract}
Introduction: When comparing oral breathing versus nasal breathing, a greater volume of air can be transported through the oral passageway but nasal breathing may also have benefits at submaximal exercise intensities. Purpose: The purpose of this study was to determine breathing efficiency during increasing levels of submaximal aerobic exercise. Methods: Nineteen individuals (males $\mathrm{N}=9$, females $\mathrm{N}=10$ ) completed a test for maximal oxygen consumption $\left(\mathrm{VO}_{2} \mathrm{max}\right)$ and on separate days 4-min treadmill runs at increasing submaximal intensities $(50 \%, 65 \%$, and $80 \%$ of $\mathrm{VO}_{2} \max$ ) under conditions of oral breathing or nasal breathing. Respiratory (respiration rate [RR], pulmonary ventilation $\left[\mathrm{V}_{\mathrm{E}}\right]$ ), metabolic (oxygen consumption $\left[\mathrm{VO}_{2}\right]$, carbon dioxide production $\left[\mathrm{VCO}_{2}\right]$ ) and efficiency measures (ventilatory equivalents for oxygen $\left[\mathrm{Veq} \cdot \mathrm{O}_{2}^{-1}\right]$ and carbon dioxide $\left[\mathrm{Veq} \cdot \mathrm{CO}_{2}^{-1}\right]$ were obtained. Data were analyzed utilizing a 2 (sex) x 2 (condition) x3 (intensity) repeated measures ANOVA with significance accepted at $\mathrm{p} \leq 0.05$. Results: Significant interactions existed between breathing mode and intensity such that oral breathing resulted in greater $\mathrm{RR}, \mathrm{V}_{\mathrm{E}}, \mathrm{VO}_{2}$, and $\mathrm{VCO}_{2}$ at all three submaximal intensities $(\mathrm{p}<.05)$. $\mathrm{Veq} \cdot \mathrm{O}_{2}{ }^{-1}$ and $\mathrm{Veq}_{2} \cdot \mathrm{CO}_{2}{ }^{-1}$ presented findings that nasal breathing was more efficient than oral breathing during the $65 \%$ and $80 \% \mathrm{VO}_{2} \max$ intensities $(\mathrm{p}<0.05$ ). Conclusion: Based on this analysis, oral breathing provides greater respiratory and metabolic volumes during moderate and moderate-to-high submaximal exercise intensities, but may not translate to greater respiratory efficiency. However when all variables are considered together, it is likely that oral breathing represents the more efficient mode, particularly at higher exercise intensities.
\end{abstract}

Keywords: Respiratory physiological processes, Musculoskeletal physiological phenomena, Running

\section{Introduction}

Respiration is a fundamental physiological process (Jones, 2001) performed during many types of activities (Romero, Coburn, Brown, \& Galpin, 2016), and there are multiple modes by which an individual can accomplish this task: through the mouth, nose, a tracheostomy tube, or combinations of these modes. In healthy individuals, respiration occurs predominantly through the nasal cavity at rest (greater than 90\%) (Camner \& Bakke, 1980) and combination of oronasal breathing during exercise (Saibene, Mognoni, Lafortuna, \& Mostardi, 1978). While strictly oral breathing will allows for greater volumetric flow at maximal exercise intensity, there may be advantages and benefits associated with different modes of breathing during submaximal work. 
Although moderate submaximal exercise does not dictate one breathing mode exclusively, it is possible that one mode (either oral or nasal) could be more efficient than the other. Investigations have been conducted at rest (Camner \& Bakke, 1980) and during maximal exercise intensity (Chinevere, Faria, \& Faria, 1999; Morton et al., 1995), but to our knowledge limited studies have been conducted examining the effect of breathing mode submaximal, and in particular, moderate-to-high intensity workloads. One such study utilizing cycle exercise at $60 \%$ of maximal oxygen consumption $\left(\mathrm{VO}_{2} \max \right)$ reported that oral breathing produced significantly higher oxygen consumption $\left(\mathrm{VO}_{2}\right)$, ventilation $\left(\mathrm{V}_{\mathrm{E}}\right)$, and respiration rate (RR) than nasal breathing (Hall, 2005). Determining the intensity at which nasal breathing begins to become a hindrance to aerobic performance and the moment to incorporate oral breathing is an area that requires further investigation.

Previous investigations determining the voluntary switch point between nasal and oronasal (combined nose and mouth) breathing have been conducted (James et al., 1997; Niinimaa, Cole, Mintz, \& Shephard, 1980; Saibene et al., 1978). The effects of nasal breathing were reported to be comfortable at lower exercise intensity, but generally at $35-41 \mathrm{~L} \cdot \mathrm{min}^{-1}$ total ventilation $\left(\mathrm{V}_{\mathrm{E}}\right)$ individuals switch from breathing nasally to oronasal breathing (Niinimaa et al., 1980; Saibene et al., 1978). While multiple studies have attempted to define the exact switching point, deviations between investigations show that there is no exact set point due to wide variance in individual breathing patterns. Additionally, the size of the nasal airway has been determined to be the greatest contributor to this switching point (Niinimaa et al., 1980), with larger nostrils and size of the nasal cavity serving to reduce resistance to airflow. An individual with a nasal airway less than $0.4 \mathrm{~cm}^{2}$ was shown to have airway impairment and an inability to sustain nasal breathing beyond the desired work capacity, which occurred in roughly $12 \%$ of the participants (Warren, Hairfield, Seaton, Morr, \& Smith, 1988).

Nasal versus oral breathing can lead to differing physiological responses in the body. Oral breathing during cycle exercise at $60 \%$ of ones aerobic capacity allows a greater volume of air to be utilized (Hall, 2005). However, nasal breathing filters pollutants from the air (Carlisle \& Sharp, 2001), and facilitates bronchial nitric oxide production, which may positively influence exercise (Bauer, Wald, Doran, \& Soda, 1994; Pepke-Zaba, Higenbottam, Dinh-Xuan, Stone, \& Wallwork, 1991; Tornberg et al., 2002; Yasuda, Itoh, Miyamura, \& Nishino, 1997). Thus, it is possible that some benefits could be conferred through nasal respiration at submaximal workloads. The purpose of the study was to determine metabolic and respiratory responses to oral versus nasal only breathing while performing treadmill running at $50 \%, 65 \%$, and $80 \%$ of the maximal aerobic capacity. We hypothesized that nasal breathing would result in lower respiratory values at the moderate $\left(65 \% \quad \mathrm{VO}_{2} \mathrm{max}\right)$ and moderate-to-high $\left(80 \% \quad \mathrm{VO}_{2} \mathrm{max}\right)$ exercise intensities. Additionally, we hypothesized that while respiratory values would be lower, when combined with the metabolic response (i.e. Veq $\cdot \mathrm{O}_{2}{ }^{-1}$, Veq $\cdot \mathrm{CO}_{2}^{-1}$ ) nasal breathing would be beneficial during submaximal exercise. We wished to provide evidence for the first time in the literature that the nasal response could be extended to work bouts greater than $60 \% \mathrm{VO}_{2} \max$.

\section{Methods}

\subsection{Subjects}

A total of 19 individuals (men $\mathrm{N}=9$, women $\mathrm{N}=10$ ) completed the protocol (Male age $=25 \pm 7 \mathrm{yr}$, height $=177.8 \pm 4.3 \mathrm{~cm}$, mass $=84.1 \pm 8.6 \mathrm{~kg}$, body fat $=16 \pm 6 \%$; Female age $=24 \pm 3 \mathrm{yr}$, height $=167.6 \pm 7.9 \mathrm{~cm}$, mass $=66.6 \pm 7.7 \mathrm{~kg}$, body fat $=27 \pm 6 \%$ ). Each participant completed the American College of Sports Medicine (ACSM) health risk questionnaire, and signed a university approved (protocol \#1311-4637) informed consent document prior to participation. The study protocol was approved by the university Biomedical Institutional Review Board in accordance with the ethical guidelines set forth in the Declaration of Helsinki.

\subsection{Procedures}

Participants were instructed to report to the Exercise Physiology Laboratory on three separate occasions. The individuals were instructed to be well hydrated, consume their last meal at least 2 hours prior to testing, refrain from caffeinated beverages for 2 hours prior to testing, and to refrain from alcoholic beverages for at least 6 hours prior to the test. Participants were asked to refrain from strenuous physical exercise during the day prior to testing. Prior to exercise testing, participant's body weight, height, and body composition were measured using a Total InBody 720 Body Composition Analyzer (InBody Co., Biospace, Seoul, Korea). Prior to the test, each participant was instructed on the use of Borg's 6-20 Rating of Perceived Exertion scale (RPE) (Borg, 1982). The graded exercise test protocol (GXT) was performed on a treadmill (Precor C954/C956, Precor Incorporated, Los Angeles, CA) with metabolic data collected using a Moxus metabolic system (AEI Technologies, Pittsburg, PA, USA) and V2 Hans Rudolph respiratory facemask (Hans Rudolph Inc., Shawnee, KS, USA). The metabolic analysis system was calibrated each day prior to testing. A heart rate monitor was applied to the participant's lower sternum under their clothing during all test sessions (Polar Electro Inc., Lake Success, NY, USA). Participants completed the progressive exercise test to the point of volitional fatigue. The first session was a $\mathrm{VO}_{2}$ max test protocol with the design to measure the voluntary switching point from oral to nasal breathing. The individual was instructed to breathe only through the nose until such time as they were not able to do so comfortably. When the participant felt they needed to "switch over" or use primarily the oral breathing mode they were instructed to raise their hand, and the $\mathrm{V}_{\mathrm{E}}$ at the moment was deemed the switching point. The $\mathrm{VO}_{2} \max$ protocol consisted of a 3-min of treadmill walking warm up at $80.47 \mathrm{~m} \cdot \mathrm{min}^{-1}(3 \mathrm{mph})$, followed by an increase in speed to $134.1 \mathrm{~m} \cdot \mathrm{min}^{-1}$ (5 mph) for one minute, and further increases in speed by $26.8 \mathrm{~m} \cdot \mathrm{min}^{-1}$ (1 $\mathrm{mph}$ ) each successive minute thereafter until test termination. On the other two sessions, participants were asked to complete a submaximal treadmill run at increasing intensities of $50 \%, 65 \%$ and $80 \%$ of their $\mathrm{VO}_{2} \max$ for 4 minutes at each intensity, once utilizing oral breathing and once with nasal breathing, performed in a counterbalanced order. Nasal only breathing was 
ensured with the use of athletic tape that was secured over the participant's mouth prior to attaching the breathing mask. Similarly, oral only breathing was ensured with the use of a swimming nose clip that was placed over the nostrils before the breathing mask was secured. Running speeds were determined using a regression equation based on the speeds and relative $\mathrm{VO}_{2}$ values obtained during the $\mathrm{VO}_{2}$ max protocol. The submaximal protocol consisted of 12 minutes of running unless the participant was not able to complete the test. After 2, 3, and 4 minutes of each stage RPE was collected.

\subsection{Statistical Analyses}

Parameters collected from the metabolic analysis unit were RR, VE, $\mathrm{VO}_{2}, \mathrm{VCO}_{2}, \mathrm{RER}, \mathrm{HR}, \mathrm{Veq} \cdot \mathrm{O}_{2}{ }^{-1}$, and $\mathrm{Veq} \cdot \mathrm{CO}_{2}{ }^{-1}$. A 2 (sex) x 2 (condition) x 3 (intensity) ANOVA with repeated measures on the last two variables test and simple main effects analysis were conducted to compare the oral and nasal breathing modes at the three exercise intensities using the SPSS (version 22, IBM Corporation, Armonk, NY) software. Significant results were determined using an alpha level of 0.05. When appropriate, post hoc analyses were completed using the paired samples t-test option in SPSS.

\section{Results}

No three-way interactions were observed for respiratory rate $(p=0.804)$, minute ventilation $(p=0.936)$, or $\mathrm{VO}_{2}$ $(p=0.817)$. There was a significant interaction between intensity and breathing mode when respiratory rate was considered $(p<0.001)$. RR was significantly greater at all exercise intensities during the oral breathing mode compared to the nasal breathing mode and the difference was magnified at the highest intensity (see Figure 1). With regards to ventilation, there was a significant interaction between intensity and breathing mode $(p<0.001)$. $\mathrm{V}_{\mathrm{E}}$ was significantly lower at each exercise intensity when participants performed nasal breathing compared to oral breathing and the difference increased as exercise became more intense (see Figure 2). Additionally, there was a significant difference between the two breathing modes for each of the three exercise intensities when oxygen uptake is considered (see Figure 3). Oxygen uptake was 8-10\% lower during the course of nasal breathing, when compared to oral breathing at any given intensity. Similarly, carbon dioxide production was 7-9\% lower during exercise performed with nasal breathing compared to the oral breathing mode $(p<0.001$, Table 1$)$.

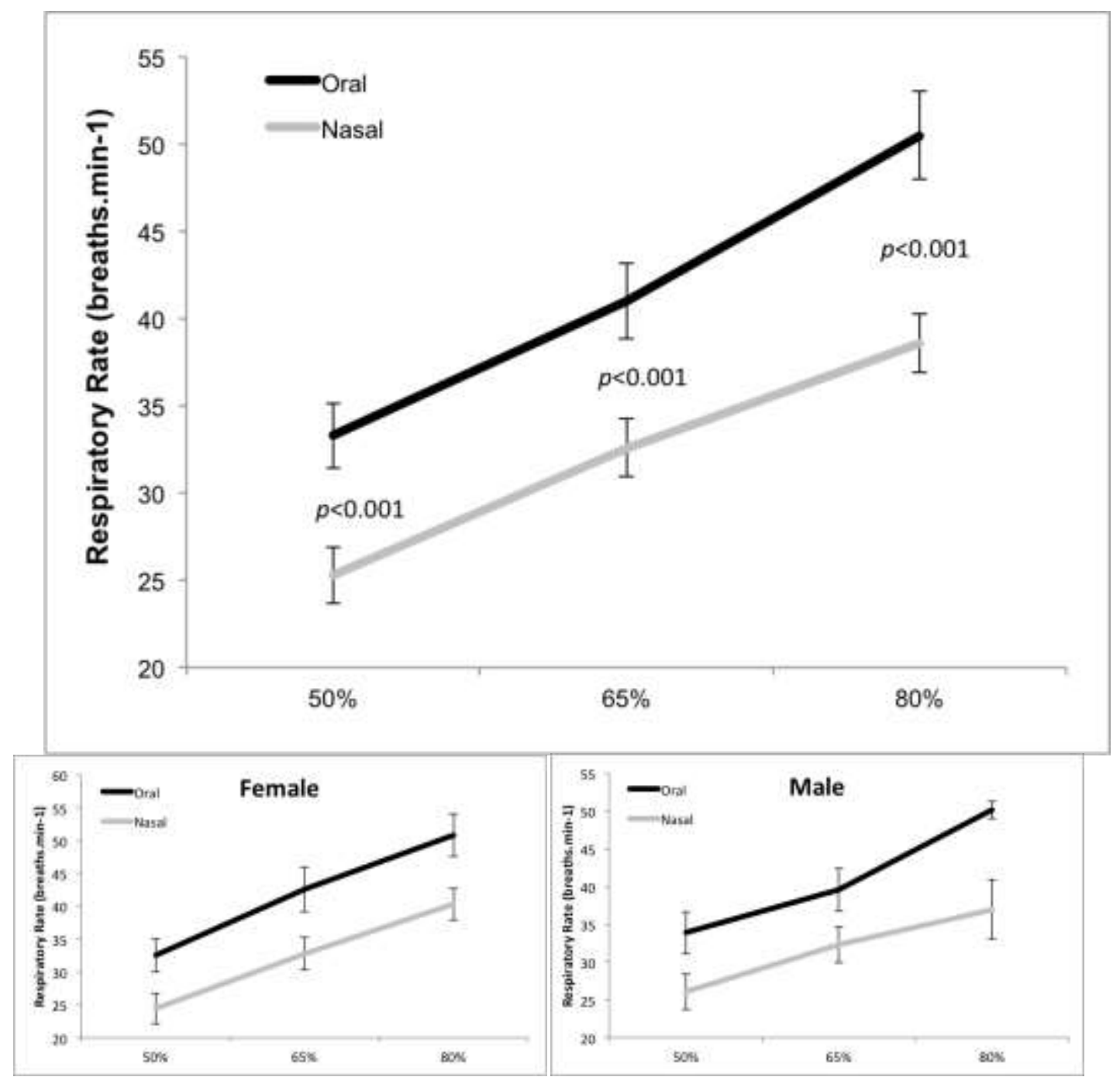

Figure 1. Respiratory rate while completing treadmill exercise under the conditions of two breathing modes (oral and nasal) across three submaximal intensities (50\%, 65\%, and 80\%). Insets represent responses separated by sex. 

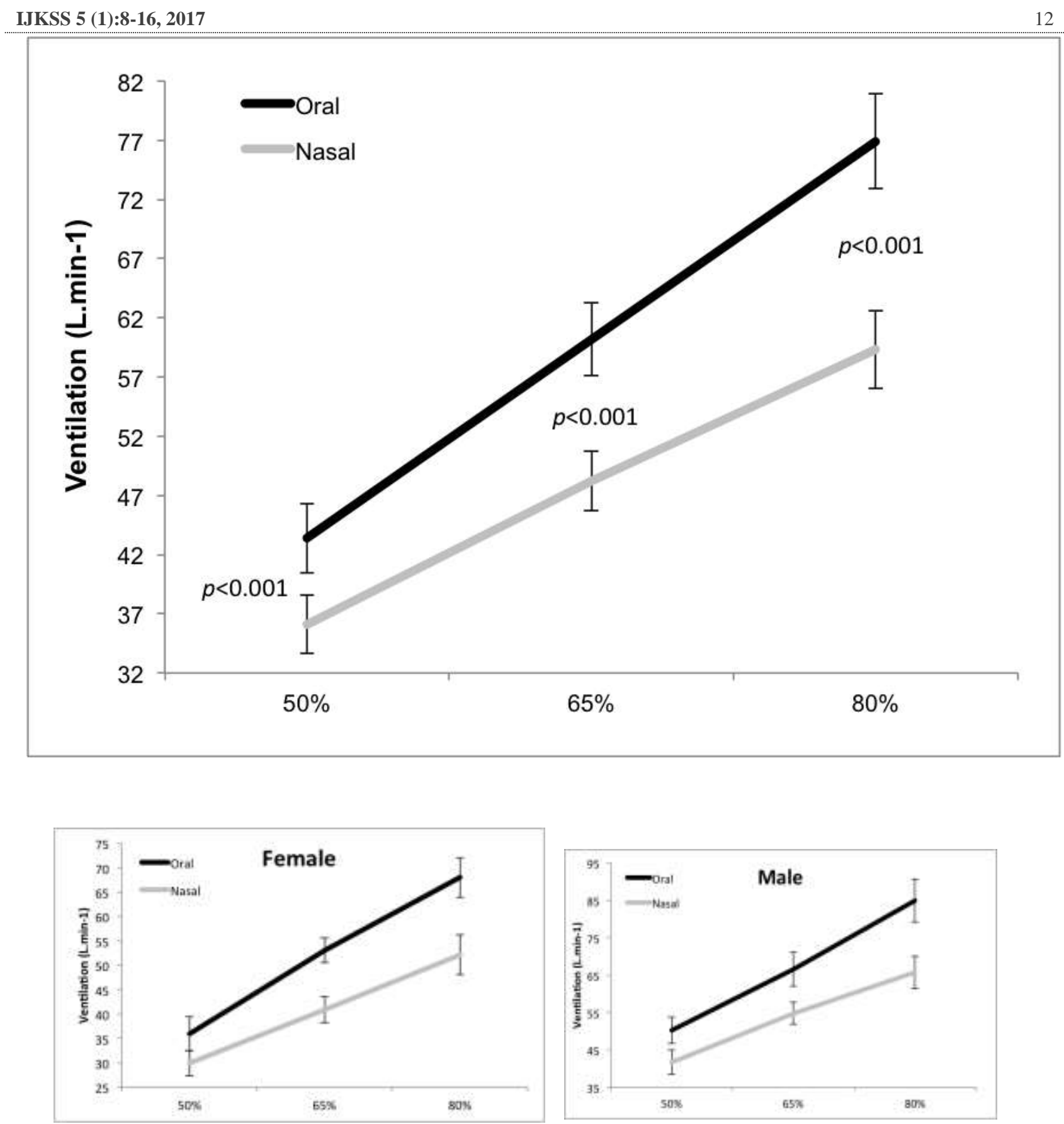

Figure 2. Ventilation rates while completing treadmill exercise under the conditions of two breathing modes (oral and nasal) across three submaximal intensities (low, moderate, and moderate-to-high). Insets represent ventilatory response separated by sex 

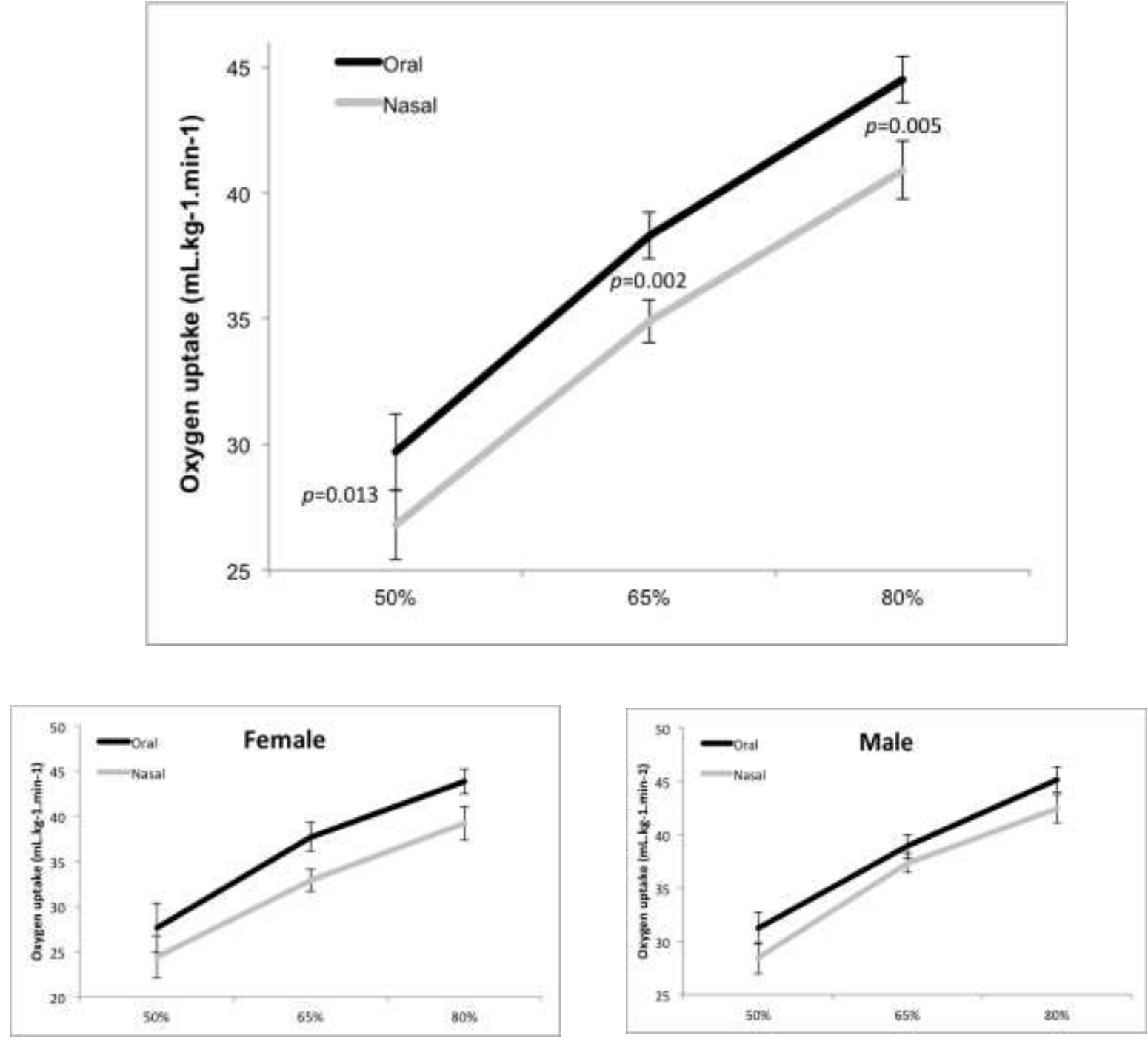

Figure 3. Relative $\mathrm{VO}_{2}$ while completing treadmill exercise under the conditions of oral and nasal breathing across three submaximal intensities $(50 \%, 65 \%$, and $80 \%)$. Insets represent oxygen consumption response separated by sex.

Table 1. Means and standard deviations of different measures between the two breathing modes at the varying intensities

\begin{tabular}{lcccccc}
\hline & & Oral & & \multicolumn{2}{c}{ Nasal } \\
Intensity & $50 \%$ & $65 \%$ & $80 \%$ & $50 \%$ & $65 \%$ & $80 \%$ \\
\hline $\mathrm{HR}\left(\mathrm{b} \cdot \mathrm{min}^{-1}\right)$ & $132 \pm 11.6$ & $157 \pm 9.7$ & $175 \pm 10.0$ & $132 \pm 13.6$ & $151 \pm 16.8$ & $166 \pm 19.8$ \\
$\mathrm{RER}$ & $0.85 \pm 0.06_{\mathrm{a}}$ & $0.92 \pm 0.04_{\mathrm{b}}$ & $0.98 \pm 0.04$ & $0.80 \pm 0.08_{\mathrm{a}}$ & $0.88 \pm 0.07_{\mathrm{b}}$ & $0.95 \pm 0.08$ \\
$\mathrm{VO}_{2}\left(\mathrm{~mL} \cdot \mathrm{min}^{-1}\right)$ & $2250 \pm 603_{\mathrm{a}}$ & $2900 \pm 534_{\mathrm{b}}$ & $3371 \pm 627_{\mathrm{c}}$ & $2051 \pm 632_{\mathrm{a}}$ & $2657 \pm 583_{\mathrm{b}}$ & $3114 \pm 704_{\mathrm{c}}$ \\
$\mathrm{VCO}_{2}\left(\mathrm{~mL} \cdot \mathrm{min}^{-1}\right)$ & $1924 \pm 573_{\mathrm{a}}$ & $2684 \pm 524_{\mathrm{b}}$ & $3296 \pm 614_{\mathrm{c}}$ & $1677 \pm 613_{\mathrm{a}}$ & $2354 \pm 629_{\mathrm{b}}$ & $2979 \pm 814_{\mathrm{c}}$ \\
$\mathrm{RPE}$ & $9 \pm 1.7$ & $12 \pm 1.8$ & $15 \pm 2.0$ & $10 \pm 2.5$ & $12 \pm 2.2$ & $15 \pm 2.3$ \\
\hline
\end{tabular}

a: Significant difference between the two breathing modes at the $50 \%$ work intensity

b: Significant difference between the two breathing modes at the $65 \%$ work intensity

c: Significant difference between the two breathing modes at the $80 \%$ work intensity

The ventilatory equivalents for oxygen and carbon dioxide were significantly higher in the oral breathing mode during the moderate and moderate-to-high two intensities, compared to the nasal breathing mode (Figure 4, Figure 5). Differences between oral and nasal breathing became greater with respect to Veq $\cdot \mathrm{O}_{2}^{-1}$ and $\mathrm{Veq} \cdot \mathrm{CO}_{2}^{-1}$ as the exercise intensity increased (Veq $\cdot \mathrm{O}_{2}^{-1} 50 \% \square=7 \%, 65 \% \square=13 \%, 80 \% \square=17 \%$, see Figure 4) (Veq. $\mathrm{CO}_{2}^{-1} 50 \% \square=1 \%, 65 \%$ $\square=8 \%, 80 \% \square=13 \%$, see Figure 5). 


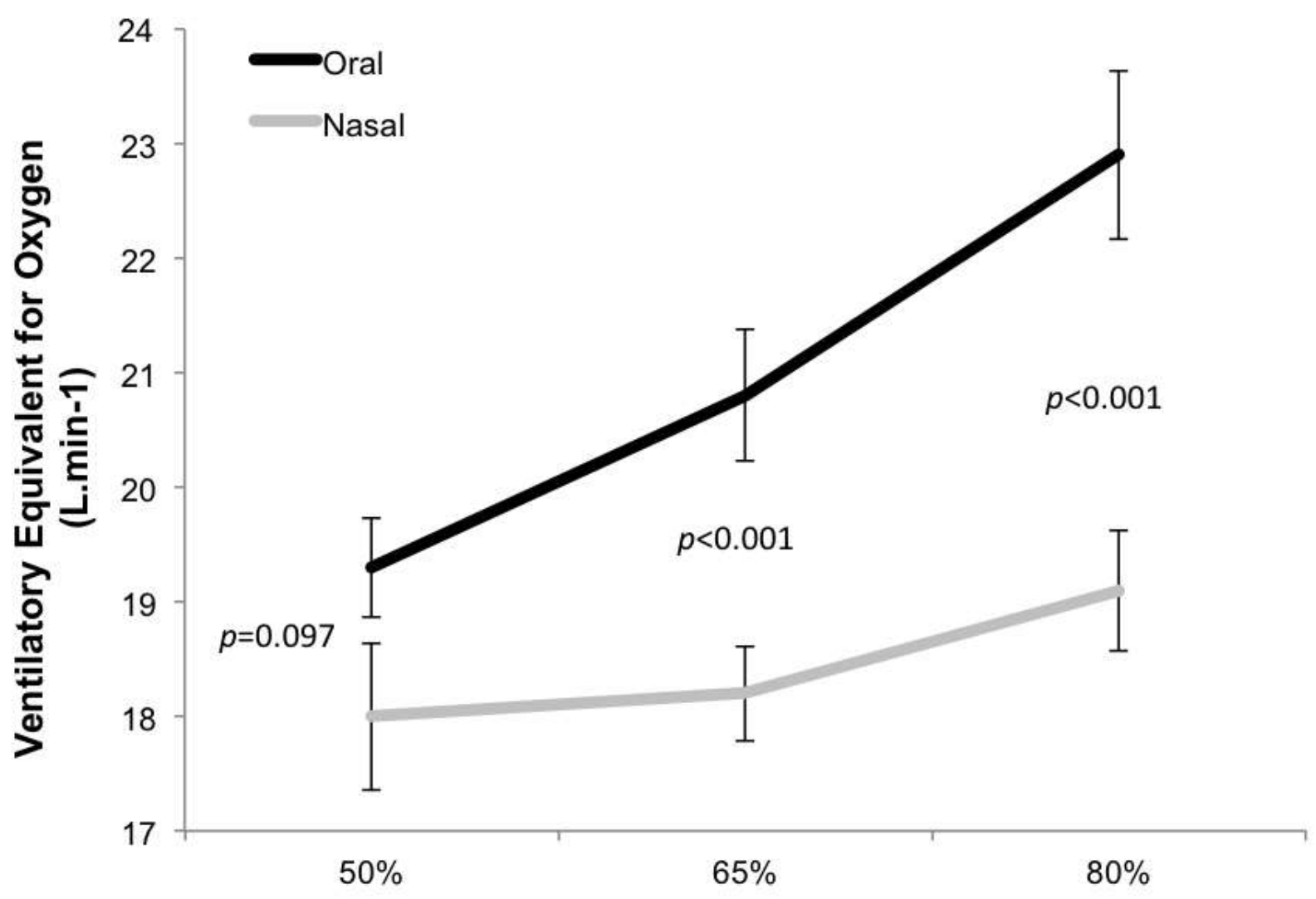

Figure 4. Ventilatory equivalent for oxygen while completing treadmill exercise under the conditions of oral and nasal breathing across three submaximal intensities $(50 \%, 65 \%$, and $80 \%)$.

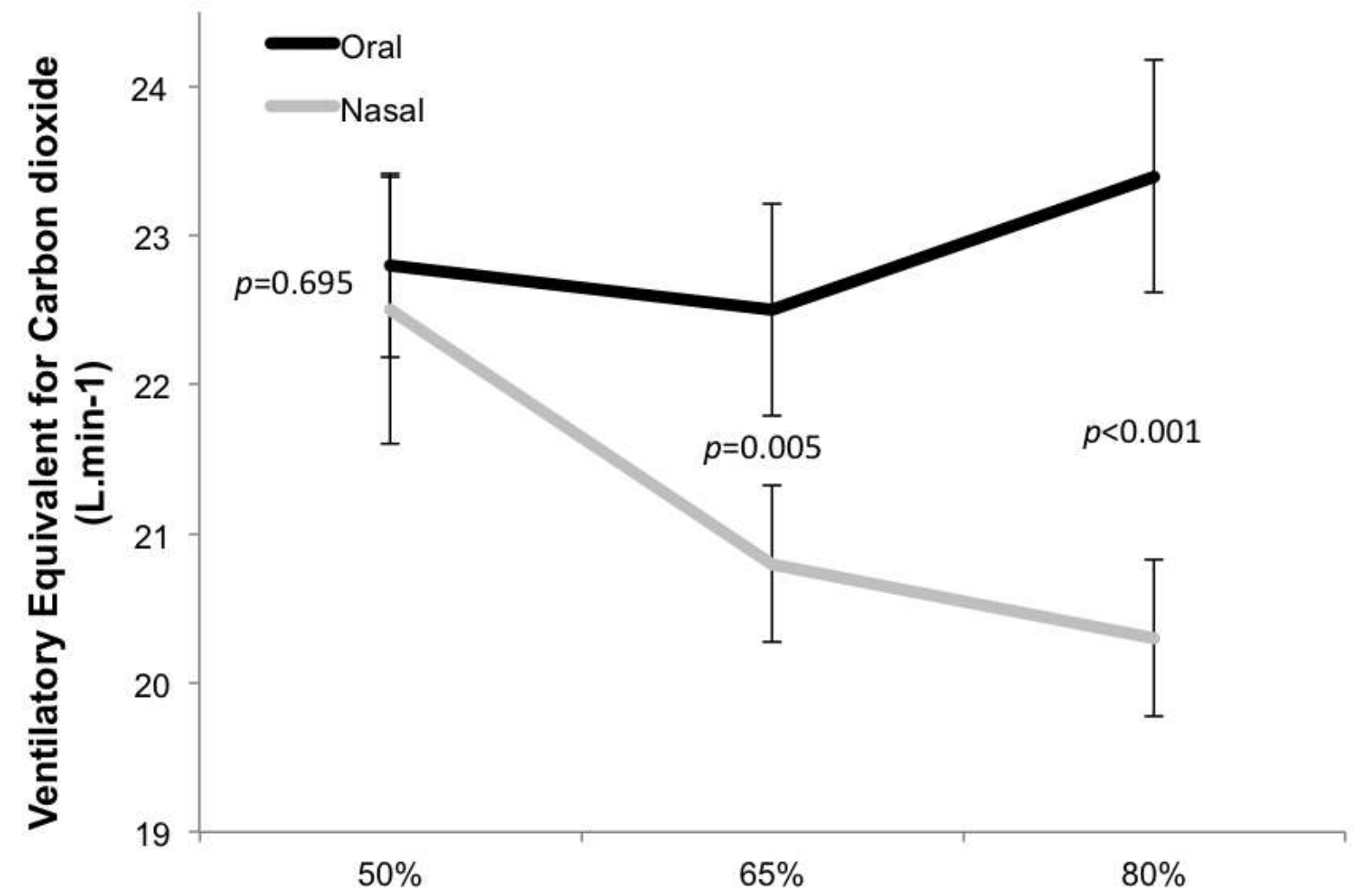

Figure 5. Ventilatory equivalent for carbon dioxide during oral and nasal breathing across three submaximal intensities (low, moderate, and moderate-to-high) 
The interaction was significant between intensity and breathing mode when RER was considered $(p<0.001)$. The findings for RER were significantly lower in the two less intense bouts $\left(50 \%\right.$ and $\left.65 \% \mathrm{VO}_{2} \max \right)$ in nasal breathing, compared to oral breathing (Table 1). The moderate-to-high exercise intensity yielded no difference with respiratory exchange ratio $(p=0.057)$. There was no significant difference between breathing modes for the dependent variables of HR $(p=0.356)$ or RPE $(p=0.586)$ during this investigation (see Table 1$)$. The mean and standard deviation for the voluntary switching point of all the participants was $52.5 \pm 14.2 \mathrm{~L} \cdot \mathrm{min}^{-1}$. Switching point for the genders was $66.2 \pm 13.7$ $\mathrm{L} \cdot \mathrm{min}^{-1}$ for males and $44.9 \pm 10.5 \mathrm{~L} \cdot \mathrm{min}^{-1}$ for females.

\section{Discussion}

The current study was carried out to investigate ventilatory efficiency with various and two breathing modes during increasing submaximal intensities of exercise. We hypothesized that nasal breathing would result in lower respiratory values at moderate and moderate-to-high exercise intensities and provide evidence for the first time in the literature that this response extends to work bouts greater than $60 \% \mathrm{VO}_{2} \mathrm{max}$. While we expected this response, we also hypothesized that when combined with the metabolic response in the form of ventilatory equivalencies, that nasal breathing would be beneficial during submaximal exercise. Other findings showed that both respiratory rate and ventilation were significantly lower at all submaximal exercise intensities for nasal breathing, and oxygen uptake displayed the same response.

In the present study, oxygen uptake was 8-10\% lower at any given exercise intensity during the nasal breathing condition when compared to oral breathing. We utilized four-minute workload stages assuming that steady state oxygen measurements would be attained. Upon further investigation, this appears to be the case for the low exercise intensity $\left(50 \% \mathrm{VO}_{2} \max \right)$ utilizing both breathing modes, and at moderate intensity $\left(65 \% \mathrm{VO}_{2} \mathrm{max}\right)$ utilizing oral breathing. It further appears that steady state oxygen values were not attained during the nasal breathing condition at moderate intensity, or at moderate-to-high intensity $\left(80 \% \mathrm{VO}_{2} \max \right)$. It is possible that provided adequate time to reach steady state in the nasal breathing condition, that oxygen uptake differences between breathing modes could have been reduced. This possibility warrants further investigation. The ventilatory equivalents for oxygen and carbon dioxide both produced significant differences between breathing modes at the moderate $\left(65 \% \mathrm{VO}_{2} \mathrm{max}\right)$ and moderate-to-high $(80 \%$ $\mathrm{VO}_{2} \max$ ) intensities. At these higher intensities, there was a significant response such that nasal breathing could be argued as the more efficient breathing mode. While a lower volume of total air was exchanged during nasal breathing (i.e. lower $\mathrm{RR}, \mathrm{V}_{\mathrm{E}}$ ) the oxygen consumed and carbon dioxide expired was sufficient to complete the work rate requirements at moderate and moderate-to-high exercise intensities. It is possible that nasal breathing could be more respiratory efficient at these intensities if an individual was willing to ventilate in that manner. While further investigations are necessary to determine an underlying mechanism for this response, a case study on a competitive triathlete who adopted a nasal-only respiration strategy during training for more than five years displayed an increase in $\mathrm{VO}_{2} \max$ and time to exhaustion while completing the same maximal workload as in an oral-only condition (Hostetter, McClaran, Cox, \& Dallam, 2016). In the current investigation nasal breathing produced lower $\mathrm{V}_{\mathrm{E}}$ at each submaximal exercise intensity, which corresponds with previous literature (Morton et al., 1995). The nose cannot transport the same volume of air as the mouth at any given exercise intensity. However it is unknown whether this limitation in $\mathrm{V}_{\mathrm{E}}$ can be made up through other respiratory or metabolic measures to make nasal breathing alone during exercise a worthy training option. The ventilatory response rose in both conditions as the exercise intensity increased, however the separation between oral $\mathrm{V}_{\mathrm{E}}$ compared to nasal was even more apparent during higher levels of exercise with a $23 \%$ difference. Another common physiological phenomenon that was observed in the present study was that respiratory rate was significantly lower during submaximal exercise in the nasal breathing condition compared to oral breathing (Morton et al., 1995). The mouth can obtain and exchange large volumes of air whereas the nasal cavity cannot accommodate the same volume due to the smaller anatomical opening. In the present investigation, respiratory rate during the nasal breathing condition was significantly lower during each submaximal exercise intensity, and this corresponds with previously published research (Chinevere et al., 1999; Hall, 2005). The moderate-to-high intensity exercise stage produced roughly 55 breaths $\cdot \mathrm{min}^{-1}$ in the oral breathing condition compared to $38 \mathrm{breaths} \cdot \mathrm{min}^{-1} \mathrm{during}$ nasal breathing. It is likely that as a greater volume of $\mathrm{CO}_{2}$ is being expired (such as in the oral breathing condition), the sensation to breathe is increased (Baraniuk \& Merck, 2008). The voluntary switching point from strictly nasal breathing to oronasal breathing was determined in the present investigation as $52.5 \mathrm{~L} \cdot \mathrm{min}^{-1}$. In the males the switching point occurred at $66.2 \mathrm{~L} \cdot \mathrm{min}^{-1}$ and in females occurred at $44.9 \mathrm{~L} \cdot \mathrm{min}^{-1}$. The voluntary switching point for females was similar to previous research (Niinimaa et al., 1980; Saibene et al., 1978) with the designated range of 35-45 L·min ${ }^{-1}$. Males generally have larger facial structures due to their larger frame, and it is possible that the nostrils and nasal cavity are also greater in anatomical volume than in females. This could allow males to have greater $\mathrm{V}_{\mathrm{E}}$ rates and inspiratory strength through the nasal cavity and result in a higher switching point than females (Hall, 2005). Further investigation and measurements are necessary to confirm this observation.

\section{Conclusions}

Nasal breathing was more efficient strictly based on the ventilatory equivalents for oxygen and carbon dioxide. However when other variables are considered together, it is likely that oral breathing represents the more effective mode, particularly at higher exercise intensities. Anecdotally, most individuals prefer to breath through the mouth at moderate-to-high intensity exercise. Nasal breathing produced oxygen uptake values that were approximately $10 \%$ lower compared to oral breathing and there are a number of possible explanations. We propose that the greatest contributing factor is the inability to reach a steady state oxygen level, but also consider that increased anaerobic energy 
production, the restriction of airflow with nasal breathing, and increased oxygen extraction at the tissue level could also play roles.

\section{References}

Baraniuk, J. N., \& Merck, S. J. (2008). Nasal reflexes: implications for exercise, breathing, and sex. Curr Allergy Asthma Rep, 8(2), 147-153. https://doi.org/10.1007/s11882-008-0025-7

Bauer, J. A., Wald, J. A., Doran, S., \& Soda, D. (1994). Endogenous nitric oxide in expired air: effects of acute exercise in humans. Life Sci, 55(24), 1903-1909. https://doi.org/10.1016/0024-3205(94)00522-2

Borg, G. A. V. (1982). Psychophysical Bases of Perceived Exertion. Medicine and Science in Sports and Exercise, 14(5), 377-381. doi: Doi 10.1249/00005768-198205000-00012

Camner, P., \& Bakke, B. (1980). Nose or mouth breathing? Environ Res, 21(2), 394-398. https://doi.org/10.1016/00139351(80)90042-0

Carlisle, A. J., \& Sharp, N. C. (2001). Exercise and outdoor ambient air pollution. Br J Sports Med, 35(4), $214-222$. https://doi.org/10.1136/bjsm.35.4.214

Chinevere, T. D., Faria, E. W., \& Faria, I. E. (1999). Nasal splinting effects on breathing patterns and cardiorespiratory responses. J Sports Sci, 17(6), 443-447. doi: 10.1080/026404199365759

Hall, R. L. (2005). Energetics of nose and mouth breathing, body size, body composition, and nose volume in young adult males and females. Am J Hum Biol, 17(3), 321-330. doi: 10.1002/ajhb.20122

Hostetter, K., McClaran, S.R., Cox, D.G., \& Dallam, G. (2016). Triathlete adapts to breathing restricted to the nasal passage without loss in VO2max or VVo2max. Journal of Sport and Human Performance, 4(1), 1-7.

James, D. S., Lambert, W. E., Mermier, C. M., Stidley, C. A., Chick, T. W., \& Samet, J. M. (1997). Oronasal distribution of ventilation at different ages. Arch Environ Health, 52(2), 118-123. doi: 10.1080/00039899709602874

Jones, N. (2001). The nose and paranasal sinuses physiology and anatomy. Adv Drug Deliv Rev, 51(1-3), 5-19. https://doi.org/10.1016/S0169-409X(01)00172-7

Morton, A. R., King, K., Papalia, S., Goodman, C., Turley, K. R., \& Wilmore, J. H. (1995). Comparison of maximal oxygen consumption with oral and nasal breathing. Aust J Sci Med Sport, 27(3), 51-55.

Niinimaa, V., Cole, P., Mintz, S., \& Shephard, R. J. (1980). The switching point from nasal to oronasal breathing. Respir Physiol, 42(1), 61-71. https://doi.org/10.1016/0034-5687(80)90104-8

Pepke-Zaba, J., Higenbottam, T. W., Dinh-Xuan, A. T., Stone, D., \& Wallwork, J. (1991). Inhaled nitric oxide as a cause of selective pulmonary vasodilatation in pulmonary hypertension. Lancet, 338(8776), 1173-1174. https://doi.org/10.1016/0140-6736(91)92033-X

Romero, B., Coburn, C. E., Brown, L. E., \& Galpin, A. J. (2016). Metabolic demands of heavy metal drumming. International Journal of Kinesiology \& Sports Science, 4(3), 32-36. doi:10.7575/aiac.ijkss.v.3n.4p.32

Saibene, F., Mognoni, P., Lafortuna, C. L., \& Mostardi, R. (1978). Oronasal breathing during exercise. Pflugers Arch, 378(1), 65-69. https://doi.org/10.1007/BF00581959

Tornberg, D. C., Marteus, H., Schedin, U., Alving, K., Lundberg, J. O., \& Weitzberg, E. (2002). Nasal and oral contribution to inhaled and exhaled nitric oxide: a study in tracheotomized patients. Eur Respir J, 19(5), 859-864. https://doi.org/10.1183/09031936.02.00273502

Warren, D. W., Hairfield, W. M., Seaton, D., Morr, K. E., \& Smith, L. R. (1988). The relationship between nasal airway size and nasal-oral breathing. Am J Orthod Dentofacial Orthop, 93(4), 289-293. https://doi.org/10.1016/08895406(88)90158-8

Yasuda, Y., Itoh, T., Miyamura, M., \& Nishino, H. (1997). Comparison of exhaled nitric oxide and cardiorespiratory indices between nasal and oral breathing during submaximal exercise in humans. Jpn J Physiol, 47(5), 465-470. https://doi.org/10.2170/jjphysiol.47.465 